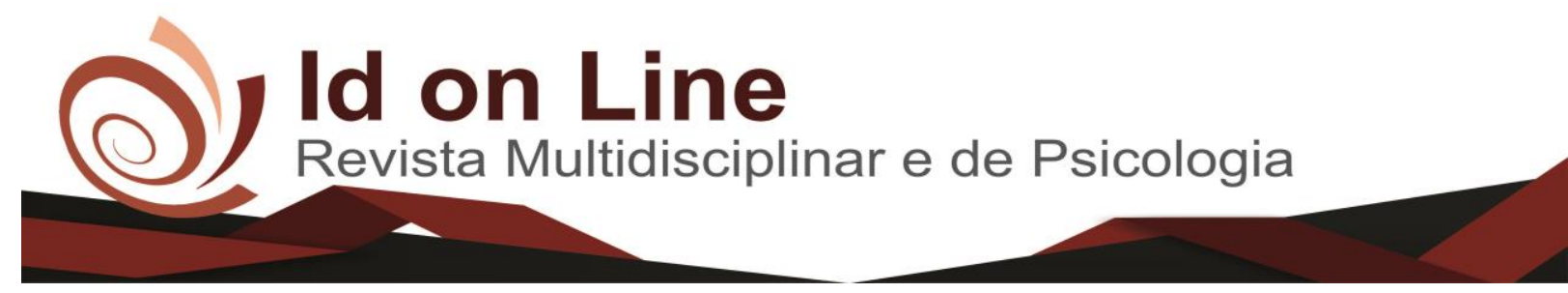

Artigo

\title{
Satisfação dos usuários com os serviços do Sistema Único de Saúde - SUS em um município do sertão de Pernambuco
}

Ially Luana de Carvalho Martins Cruz ${ }^{1}$; Alaine Santos Parente ${ }^{2}$;

Fabiola Olinda de Souza Mesquita ${ }^{3}$; Aline Silva Jerônimo ${ }^{4}$

Resumo: A satisfação do usuário é considerada como diretriz orientadora para que os gestores de saúde programem melhorias dos serviços públicos. O presente trabalho tem como objetivo identificar a satisfação dos usuários dos serviços públicos de saúde no município de Salgueiro PE, acerca do acolhimento, resolutividade, acesso e tempo de espera para atendimento. Foi realizado estudo descritivo de abordagem quantitativa. 56,41\% considerou o acolhimento bom; para $84,62 \%$ o serviço é resolutivo; $61,54 \%$ considera o acesso bom; $56,41 \%$ mostraram-se insatisfeitos com o tempo para atendimento. A maior parte dos usuários mostrou-se satisfeitos com os serviços de saúde municipais.

Palavras-chaves: Satisfação do paciente. Qualidade da assistência à Saúde. Serviços de saúde.

\section{Satisfaction of users with Health Unic System - SUS services in a municipality in the hinterland of Pernambuco}

\begin{abstract}
User satisfaction is considered as directive for health managers to program improvement of public services. The present study had as objective identify the satisfaction of users of public health services in Salgueiro PE municipality, about the host, resoluteness, access and waiting time for care. A descriptive study was carried out with a quantitative approach. $56.41 \%$ considered good host ; $84.62 \%$ for the service is resolute ; $61.54 \%$ consider good access ; $56.41 \%$ were shown to be dissatisfied with the time to care. Most users showed their satisfaction with the municipal health services.
\end{abstract}

Keywords: Patient satisfaction. Quality of health care. Health services.

\section{Introdução}

O movimento brasileiro de Reforma Sanitária, na década de 70, foi marcado por diversas mobilizações e lutas visando o acesso universal a um sistema de saúde público inclusivo.

\footnotetext{
${ }^{1}$ Farmacêutica. Residência em Saúde Coletiva com ênfase em gestão de redes pela Universidade de Pernambuco. Contato: iallyluana@yahoo.com.br;

${ }^{2}$ Fisioterapeuta. Residência em Saúde Coletiva com ênfase em gestão de redes pela Universidade de Pernambuco; Contato: alainefisio@ hotmail.com;

${ }^{3}$ Odontóloga. Especialista em Gestão em Saúde pela Universidade Federal do Vale do São Francisco (UNIVASF).

Contato: fabiolaolinda@yahoo.com.br;

${ }^{4}$ Mestre em Ciências da Saúde pela Universidade Federal do Vale do São Francisco. Contato: alainefisio@hotmail.com
} 
Com a constituição de 1988, a saúde foi assegurada como direito individual a cada cidadão, determinando que as ações e serviços de saúde fossem organizados em consonância com os princípios da universalidade do acesso, integralidade da assistência à saúde e a equidade no contexto de um Sistema Único de Saúde (SUS) (MITRE; ANDRADE; COTTA, 2012).

Através da aprovação da Lei Orgânica de Saúde n 8.080 em 1990 o SUS foi regulamentado. A lei veio definir o modelo operacional, a forma de organização e o funcionamento do SUS, além de seus princípios e diretrizes, que garantem a população acesso aos serviços de saúde e a participação da população no processo de formulação, execução e avaliação das políticas de saúde por meio da garantia da participação popular (PIOVESAN et al., 2014).

De acordo com Andrade (2015a, 2015b) a criação do SUS transferiu ao Estado a incumbência de assegurar e financiar todos os serviços de saúde à população brasileira. Desde então, as políticas públicas de saúde passaram a ser elaboradas de forma a garantir oferta eficiente e equânime, resultando em melhorias no acesso aos serviços de saúde. Dentre os vários avanços proporcionados pelo SUS destaca-se a Estratégia Saúde da Família (ESF) considerada um mecanismo de reorientação do modelo assistencial de atenção primária a saúde visando viabilizar e organizar a Atenção Primária em Saúde.

A Estratégia de Saúde da Família surge da perspectiva de mudança da organização do trabalho, do processo saúde-doença e do modo de cuidar. Compromete-se a garantir assistência ao usuário na sua integralidade, considerando seu contexto familiar e social. Apresenta papel primordial no que diz respeito as ações preventivas, de promoção da saúde, atendimento, monitoramento e acompanhamento das famílias, considerando os indicadores epidemiológicos. Tem como foco a coletividade, desenvolvendo ações que venham a atender as demandas da população, garantindo acesso aos serviços de saúde e a qualidade das ações, ordenados pelos princípios da integralidade, coordenação e longitudinalidade (ANDRADE et al., 2015a; LIMA et al ., 2014).

A Portaria n ${ }^{\circ}$ 2488/GM/MS, de 21 de outubro de 2011 aprova a Política Nacional de Atenção Básica, estabelecendo a revisão de diretrizes e normas para a organização da Atenção Básica, para a Estratégia Saúde da Família e o Programa de Agentes Comunitários de Saúde (PACS), que tem na Saúde da Família sua estratégia prioritária para expansão e consolidação 
da atenção básica (BRASIL, 2011).

Essa organização consolidou o direito de participação nas tomadas de decisões dos segmentos: gestores, prestadores de serviços, trabalhadores de saúde e usuários. Esses se tornaram elementos importantes na definição de políticas e na execução das ações e serviços de saúde, estimulando a comunidade a exercer sua cidadania com a participação e controle social na área de saúde (MARTINS; SANTOS, 2012; BESERRA; COSTA; OLIVEIRA, 2014).

Nesse sentido, ressalta-se a importância da participação popular e o controle social como um dos princípios do SUS de maior relevância política, determinante fundamental para o exercício da cidadania. Esses garantem que a população participe ativamente no processo de formulação das políticas públicas de saúde e do controle sobre a ação do Estado. A participação da comunidade na rotina dos serviços de saúde como no planejamento, execução e avaliação das ações e serviços deve ser estimulada, bem como o conhecimento que interfere diretamente na tomada de decisões e permite que a população organizada possa exercer seu papel de fiscalizador e avaliador dos serviços de saúde (COELHO, 2012; CRUZ; ROLIM; SAMPAIO, 2013).

O principal elemento legal responsável por institucionalizar a participação social no SUS foi a Lei 8.142/1990 que assegurou a participação da sociedade nas esferas deliberativas representadas pelos conselhos de saúde, onde foi possível um maior controle das políticas e execução dos serviços pela sociedade, além de garantir autonomia nas decisões pertinentes à saúde (SANTOS; JUNIOR; LELES, 2013).

Com a consolidação do controle social no SUS foram introduzidos, a partir da década de 90 no Brasil, os estudos sobre satisfação dos usuários dos serviços de saúde, o que permitiu uma maior participação da comunidade, uma vez que a qualidade dos serviços passa a ser avaliada a partir da percepção do usuário e através das ações prestadas para posteriormente essa avaliação promover resultados promissores acerca da qualidade dos serviços (FERRO et al., 2015).

A avaliação da satisfação do usuário é um importante instrumento para analisar a qualidade dos serviços e ações de saúde. A opinião do mesmo é de grande importância para melhor adequar os serviços de saúde a população, desde a estrutura física até a qualidade da assistência prestada dos estabelecimentos de saúde. Portanto, a satisfação do usuário de saúde é diretriz orientadora a fim de que gestores e equipe de saúde a utilize para implementar 
melhorias dos serviços oferecidos a população. A percepção dos usuários, com relação à qualidade dos serviços públicos prestados funciona como indicador de avaliação e monitoramento para profissionais e gestores, a fim de que possam adaptar os seus serviços às necessidades da população (FADEL; FILHO, 2009; RAIMUNDO; RODRIGUES; SILVA, 2010; FERRO et al., 2015).

Um desafio bastante enfrentado pelos gestores diz respeito à qualidade dos serviços de saúde, onde satisfazer as necessidades dos usuários é o ponto chave a ser resolvido. Contudo, avaliar a satisfação dos usuários, em relação à percepção que o mesmo tem sobre o serviço pode ser considerado como um componente da avaliação da qualidade (CASTRO, 2008; PISCO, 2006).

Desse modo, a pesquisa objetivou identificar a satisfação dos usuários dos serviços públicos de saúde existentes no município de Salgueiro- PE, permitindo compreender a visão do usuário em relação ao acolhimento, grau de resolutividade, acesso e tempo de espera para atendimento.

\section{Metodologia}

Trata-se de um estudo descritivo- exploratório. A modalidade de pesquisa exploratória tem como objetivo caracterizar o problema, classificar e definir o mesmo.

Para Rodrigues (2007) na abordagem descritiva as variáveis são analisadas sem que haja interferência do pesquisador, utilizando-se de técnicas padronizadas de coleta de dados, como questionários. Quanto à forma de abordagem, trata-se de uma pesquisa quantitativa, onde as opiniões são traduzidas em números, sendo analisadas e classificadas utilizando técnicas estatísticas. Foi utilizada a pesquisa estatística descritiva simples através de exposição do percentual das respostas obtidas.

O estudo foi realizado no município de Salgueiro PE, nas 12 (doze) Unidades Básicas de Saúde (UBS), localizadas na zona urbana, que tem como modelo de atenção a Estratégia Saúde da Família. Foram excluídas as unidades da zona rural pelo difícil acesso e falta de transporte para locomoção.

O público alvo da pesquisa incluiu todos os usuários presentes nas Unidades de Saúde no momento da coleta de dados com idade igual ou superior a 18 anos. Foi utilizado como 
instrumento para coleta de dados um formulário estruturado, composto por 28 questões de múltipla escolha. As variáveis estudadas foram: 1) Perfil socioeconômico (grau de instrução, cor, renda); 2) Acesso; 3) Acolhimento; 4) Resolutividade; 5) Tempo de espera.

A coleta dos dados foi realizada entre os meses de abril a maio de 2016. Ao final do levantamento, os dados foram processados, analisados e calculados em percentuais a partir de cada parâmetro utilizado no questionário e esses, inseridos em gráficos e tabelas, aplicados no programa Microsoft Excel 2007® e Microsoft Word 2007®, respectivamente.

Foram respeitados todos os preceitos éticos baseando-se na Resolução nº. 466/2012 do Conselho Nacional de Saúde que trata dos aspectos éticos da pesquisa envolvendo seres humanos e as atividades só foram iniciadas após a aprovação pelo mesmo (BRASIL, 2012). Por razões de segurança e confidencialidade foram apresentados os objetivos e relevância do estudo aos entrevistados, além dos demais aspectos éticos aos sujeitos da pesquisa, formulados através de um Termo de Consentimento Livre e Esclarecido (TCLE). Após explicação sobre a natureza da pesquisa, seus objetivos e métodos, autorizando sua participação voluntária, os sujeitos assinaram o TCLE.

O projeto foi submetido ao Comitê de Ética em Pesquisa da Universidade de Pernambuco sob o parecer $n^{\circ} 1.498 .634$ tendo sido aprovado e garantido pelo pesquisador o total sigilo e anonimato dos dados coletados e trabalhados.

\section{Resultados e discussão}

Conforme descrito na metodologia as variáveis analisadas foram: 1) Perfil socioeconômico; 2) Acesso; 3) Acolhimento; 4) Resolutividade; 5) Tempo de espera. Foram entrevistados 78 usuários, com idade entre 18 e 65 anos. Destes, 76,92\% eram do gênero feminino, 43,59\% não haviam completado o $1^{\circ}$ grau, $66,67 \%$ se declararam da cor parda e $73,08 \%$ declararam renda familiar até 1,5 salário mínimo (Tabela 1). 
Tabela 1 - Perfil socioeconômico e demográfico dos usuários das Unidades de Saúde da Família do município de Salgueiro-PE, 2016.

\begin{tabular}{lcc}
\hline Gênero & $\mathbf{N}$ & $\mathbf{\%}$ \\
\hline Feminino & 60 & 76,92 \\
Masculino & 18 & 23,08 \\
Grau de instrução & 34 & 43,59 \\
$1^{\circ}$ grau incompleto & 6 & 7,69 \\
$1^{\circ}$ grau completo & 9 & 11,54 \\
$2^{\circ}$ grau incompleto & 19 & 24,36 \\
$2^{\circ}$ grau incompleto & 3 & 3,85 \\
Superior incompleto & 5 & 6,41 \\
Superior completo & & \\
Cor & 18 & 23,08 \\
Branca & 5 & 6,41 \\
Preta & 2 & 2,56 \\
Amarela & 52 & 66,67 \\
Parda & 1 & 1,28 \\
Indígena & & \\
Renda Familiar & 57 & 73,08 \\
Até 1,5 salário mínimo & 13 & 16,66 \\
De 1,5 a 3 salários mínimos & 5 & 6,41 \\
De 3 a 4,5 salários mínimos & 3 & 3,85 \\
De 6 a 10 salários mínimos & & \\
\hline
\end{tabular}

Fonte: Pesquisa direta, 2016.

A situação socioeconômica e a escolaridade são importantes determinantes do uso de serviços de saúde. Espera-se que entre os usuários do SUS, predomine a baixa escolaridade e/ou a baixa renda (LIMA, MATOS, CAMARANO, 2006). Na pesquisa de Costa e Loyola (2008) também houve predominância de usuários com $1^{\circ}$ grau incompleto (67\%). Dados semelhantes foram apontados por Gouveia et al. (2011), revelando que 70,6\% dos usuários não chegaram a concluir o $1^{\circ}$ grau. Aponta também maior frequência da população feminina entre os usuários do SUS $(58,97 \%)$.

Segundo Bós (2004), indivíduos com um nível maior de educação teriam conhecimento superior sobre a sua situação de saúde e, em geral, melhores condições socioeconômicas, possibilitando-o de buscar alternativas de atendimento, incluindo os que são pagos. Os resultados desse trabalho corroboram com essa hipótese.

No que diz respeito ao acesso, observou-se que $80,77 \%$ dos atendimentos são realizados mediante agendamento de consulta, onde a maioria dos usuários 56,41\% relataram 
que esses ocorrem em dia e horário específico da semana. Quando questionado sobre o grau de dificuldade para se chegar até a unidade, 75,64\%consideraram ser fácil, corroborando com a pesquisa de Corradi (2014), onde $92 \%$ dos entrevistados consideraram fácil o acesso ao serviço.

A limitação do acesso quando decorrente da precariedade da organização da rede de serviços dificulta a realização do cuidado e a utilização dos recursos disponíveis, fazendo com que muitas vezes ocorra uma piora ou até mesmo cronificação do problema de saúde do usuário (GRDEN et. al., 2015). O reconhecimento do acesso como um dos princípios do SUS estimula a procura por melhores resultados nas intervenções em saúde, tornando a continuidade do cuidado algo essencial a ser reproduzido em todos os níveis da assistência a saúde, almejando, dessa forma, a qualificação do cuidado (CORRÊIA et. al., 2011).

$\mathrm{Na}$ pesquisa realizada por Trad et. al. (2002), os participantes relataram que a localização das Unidades de Saúde da Família é considerada, na maioria dos municípios, de fácil acesso. Fato considerado importante se relacionado à satisfação dos usuários, pois a mesma está associada à facilidade de acesso ao profissional e às ações de saúde.

No que se refere ao acesso ao serviço, $61,54 \%$ consideraram como bom, enquanto quase $20 \%$ classificaram como ruim (Tabela 2 ).

Tabela 2 - Acesso dos usuários as Unidades de Saúde da Família no município de Salgueiro-PE, 2016.

\section{Como é o acesso ao atendimento de consulta ao serviço de} saúde?

Agendamento

Demanda Espontânea

$\mathrm{N} \quad \%$

\section{$\%$}

A marcação da consulta na unidade de saúde pode ser feita:

Em qualquer dia e horário da semana

Em qualquer dia da semana, em horário específico

Dias específicos fixos, em qualquer horário

Dias específicos fixos, em horários específicos

63

15

80,77

19,23

18

11

5

44
23,08

14,10

6,41

56,41

Para se chegar a unidade de saúde é:

Muito fácil 1

Fácil 59 75,64

Razoável

$4 \quad 5,13$

Difícil

14 17,95 
O acesso ao serviço é:

$\begin{array}{lcc}\text { Excelente } & 8 & 10,26 \\ \text { Bom } & 48 & 61,54 \\ \text { Ruim } & 15 & 19,23 \\ \text { Péssimo } & 7 & 8,97\end{array}$

Fonte: Pesquisa direta, 2016.

Pires (2010) acredita haver iniquidades no acesso e ineficiência no sistema de saúde quanto aos usuários do SUS. Isso está associado à desarticulação entre a oferta dos serviços e as demandas trazidas pela população, amparada na dificuldade dos profissionais em escutar as necessidades de saúde da população. O acesso à saúde deve extrapolar a assistência em si, sendo a acessibilidade aos diversos níveis de complexidade dos serviços fundamental para a concretização desse direito universal, no momento certo e de maneira rápida (CORRÊA et al 2011).

Assunção (2006) afirma que as longas filas, as condições precárias de atendimento e o tratamento desumano da população, frequentemente divulgado pela mídia, corroboram para denegrir a imagem das instituições públicas de saúde. Parente (2000) ressalta que, à medida que são apresentados melhores serviços, as expectativas dos clientes são elevadas. Isso faz com que a melhoria dos serviços prestados acarrete aumento no nível de exigências e das expectativas. Em consequência, o aumento das expectativas estimula os prestadores de serviços a prosseguirem em seus esforços para a melhoria contínua.

Em relação ao acolhimento, a maior parte dos usuários apontou como sendo bom $56,41 \%$; enquanto $84,62 \%$ afirmaram ter recebido tratamento respeitoso por parte dos profissionais do serviço (Tabela 3).

Tabela 3 - Acolhimento dos usuários nas Unidades de Saúde da Família no município de Salgueiro-PE, 2016.

\begin{tabular}{lcc}
\hline O acolhimento no momento da chegada ao serviço é: & N & \% \\
\hline Excelente & 8 & 10,26 \\
Bom & 44 & 56,41 \\
Ruim & 21 & 26,92 \\
Péssimo & 5 & 6,41
\end{tabular}


Recebeu tratamento respeitoso pelos profissionais?

Sim

Não

Fonte: Pesquisa direta, 2016.

O acolhimento é visto como uma ferramenta, estratégia e arranjo tecnológico utilizado para iniciar mudanças no processo de trabalho em saúde, garantir o acesso em serviços humanizados, resolutos e de qualidade com a responsabilização coletiva dos trabalhadores às necessidades dos usuários (MITRE, 2012).

Constitui-se em instrumento potente para a reorganização da Atenção à saúde na Saúde da Família, podendo legitimar a equipe como "porta de entrada" na medida em que consegue influenciar positivamente no padrão de utilização dos serviços por parte dos indivíduos, além de intervir em questões sociais e organizacionais do serviço (CHAGAS, VASCONCELLOS, 2013).

Para Gouveia et. al. (2009), os usuários do Programa de Saúde da Família expressam um elevado grau de satisfação em relação à dimensão relacional no que se refere ao acolhimento e gentileza por parte dos profissionais da equipe; portanto, em relação à qualidade dos profissionais de saúde.

A interação entre profissionais de saúde e usuários é um fator fundamental para garantia da efetividade do cuidado, continuidade do tratamento e a forma como o usuário consegue acessar o serviço, bem como o tratamento recebido e o acolhimento vão interferir no estado de saúde do indivíduo e da coletividade (RAMOS; LIMA, 2003).

O acolhimento não se limita apenas a uma recepção cordial, mas extrapola esse conceito, incluindo a escuta ativa do usuário. Aspectos como vínculo, resolutividade e desempenho profissional estão relacionados com o acolhimento. A relação profissionalpaciente é fortalecida quando se tem um atendimento humanizado, proporcionando ao paciente segurança e contribuindo na melhoria de saúde do mesmo (BRASIL, 2004).

No quesito da resolutividade das Unidades de Saúde da Família, quando questionados se existe garantia de atendimento conforme a gravidade do caso, 65,38\% dos usuários afirmaram que sim. Para $84,62 \%$ os serviços de saúde prestados estão resolvendo os problemas e necessidades da população, embora 51,28\% relataram que já precisaram ser 
encaminhados para outros serviços que compõem a rede de assistência a saúde do município (Tabela 4).

Tabela 4 - Resolutividade das Unidades de Saúde da Família do município de SalgueiroPE, 2016.

\begin{tabular}{lcc}
\hline $\begin{array}{l}\text { Caso haja consulta de urgência, há garantia de atendimento } \\
\text { conforme a gravidade do problema? }\end{array}$ & N & \% \\
\hline Sim & 51 & 65,38 \\
Não & 27 & 34,62 \\
Seu problema foi resolvido? & 66 & 84,62 \\
Sim & 12 & 15,38 \\
Não & & \\
Precisou ser encaminhado para outro serviço? & 40 & 51,28 \\
Sim & 38 & 48,72 \\
Não & & \\
\hline
\end{tabular}

Fonte: Pesquisa direta, 2016.

A resolutividade dos serviços de saúde é uma maneira de avaliar os serviços a partir dos resultados obtidos do atendimento ao usuário. A resolutividade dos serviços no modelo hierarquizado por níveis de atenção pode ser avaliada por dois modos. Quando ocorre dentro do próprio serviço, quanto a capacidade de atender a sua demanda e de encaminhar os casos que necessitam de atendimento mais especializado e, dentro do sistema de saúde, que vai desde a consulta inicial do usuário na Unidade de Saúde da Família até a resolução de seu problema em outros níveis de atenção à saúde (TURRINI, LEBRÃO, CESAR, 2012).

As pesquisas de satisfação dão ênfase aos diferentes aspectos envolvendo a assistência à saúde, que vão desde a relação médico-paciente, até a estrutura das instalações e qualidade do trabalho dos profissionais. No que diz respeito à participação do usuário, a satisfação está ligada à maior apropriação no uso dos serviços de saúde, levando em consideração tanto a relação do usuário ao processo do cuidado, quanto à estrutura das instalações (ANDRADE; VAITSAMN, 2005; ESPERIDÃO; TRAD, 2005).

Sobre o tempo de espera entre o agendamento e a consulta, verificou-se que para $46,16 \%$ dos usuários o tempo de espera correspondia a 1 dia; $37,18 \%$ menos de 1 semana e 16,66\% espera acima de 2 semanas para atendimento na unidade. Quando questionados em 
relação a satisfação com o tempo de espera, 56,41\% mostraram-se insatisfeitos. Ressalta-se que as consultas em questão foram médicas, odontológicas e de enfermagem.

Tabela 5 - Tempo de espera para atendimento nas Unidades de Saúde da Família no município de Salgueiro- PE, 2016.

\begin{tabular}{lcc}
\hline $\begin{array}{l}\text { Qual o tempo de espera entre o agendamento da consulta e o dia } \\
\text { de atendimento na USF? }\end{array}$ & $\mathbf{N}$ & $\%$ \\
\hline 1 dia & 36 & 46,16 \\
Menor que 1 semana & 29 & 37,18 \\
Maior que 2 semanas & 1 & 1,28 \\
1 mês & 8 & 10,26 \\
Menor que 1 mês & 2 & 2,56 \\
Meses & 2 & 2,56 \\
Ficou satisfeito com o tempo de espera para ser atendido? & & \\
Sim & 34 & 43,59 \\
Não & 44 & 56,41 \\
\hline Font Pesquida
\end{tabular}

Fonte: Pesquisa direta, 2016.

A insatisfação com o tempo de espera para atendimento é vista de maneira rotineira nos serviços de saúde, indo de encontro com o resultado encontrado no estudo de Andrade e Ferreira (2006). Outros estudos evidenciam a espera para o atendimento como sendo o maior problema apontado pelos usuários, como visto na pesquisa de Franco e Campos (1998).

Em relação à satisfação com o atendimento recebido nas Unidades de Saúde da Família, os usuários se posicionaram em sua grande maioria de maneira positiva, embora um percentual considerável não se mostraram satisfeitos. O percentual de $38,46 \%$ declarou-se muito satisfeito com o atendimento recebido; $19,23 \%$ estavam satisfeitos; $33,34 \%$ afirmaram estarem pouco satisfeitos, enquanto 8,97\% insatisfeitos (Gráfico 1). 
Gráfico 1: Percentual de satisfação dos usuários com o atendimento recebido nas Unidades de Saúde da Família no município de Salgueiro- PE, 2016.

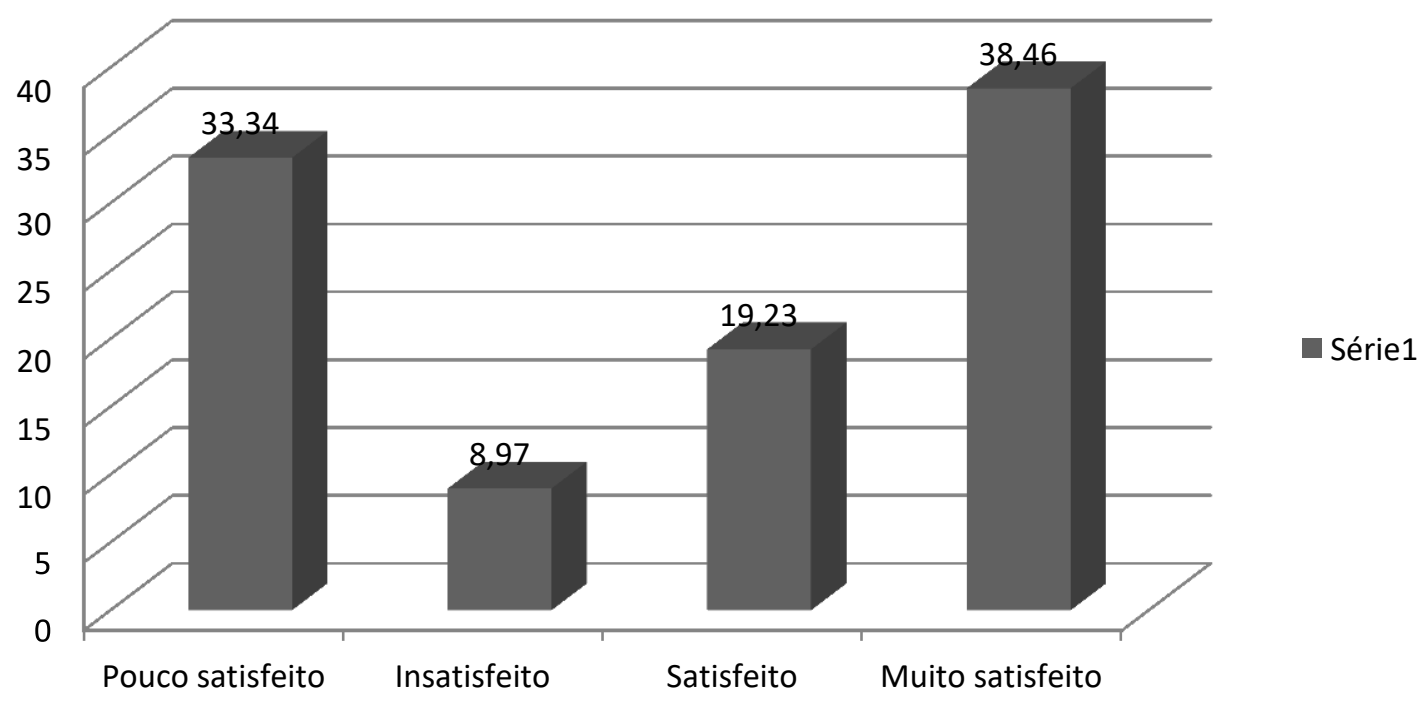

Fonte: Pesquisa direta, 2016.

Apesar de alguns resultados não se apresentarem tão favoráveis, o usuário mostrouse satisfeito com os serviços públicos de saúde oferecidos. Assim, fica evidenciado que a satisfação do usuário deve ser o objetivo final de todo serviço de saúde, devendo ser considerado alguns pontos importantes no momento da chegada do usuário ao serviço, como a percepção, que é decorrente de fatores distintos, como econômicos, sociais, culturais, condição de vida, além da condição pessoal de cada um no momento da entrevista.

A pesquisa de Guedes e Garcia (2001) mostra que as respostas dos usuários acerca do nível de satisfação com os atendimentos nos serviços públicos tendem para um direcionamento favorável, pois existe um receio por parte dos participantes que as respostas negativas podem gerar algum tipo de punição.

Em contextos muito desiguais, como é caso do Brasil, alguns fatores influenciam diretamente na satisfação dos usuários, como a estrutura, a organização dos serviços de saúde e os modelos assistenciais vigentes. Quando existe grande dificuldade em acessar determinado serviço por uma população específica, no momento que se é atendido, já é motivo de grande satisfação, pois, em geral, a população não tem expectativas positivas com relação às instituições públicas (ANDRADE; VAITSAMN, 2005; GOUVEIA et al., 2011). 
As pesquisas de satisfação não refletem de forma objetiva como está sendo o tratamento das pessoas nos estabelecimentos de saúde. Com isso, a tese de que a percepção do cuidado é motivada pela subjetividade do conceito de qualidade e pela expectação do usuário é acentuada. O grau de satisfação dos usuários envolve aspectos dos serviços, como: qualidade, estrutura física e organização, acesso, bem como os aspectos ligados a relação entre o médico e o paciente (ESPERIDÃO; TRAD, 2005; KAWABATA; MURRAY; VALENTINE, 2001). Observa-se, a partir destes resultados, a relevância da qualidade do atendimento aos usuários como determinante do seu nível de satisfação, evidenciando a necessidade de o serviço qualificar, cada vez mais, o processo de atendimento ao paciente.

No contexto da qualidade em saúde, avaliar é diagnosticar uma realidade a fim de nela intervir para que os diferentes serviços de saúde cumpram padrões mínimos de qualidade. Nesta conjuntura de avaliação de serviços de saúde se evidencia que qualidade não é mais considerada como algo facultativo, mas como fator primordial para permanência no mercado e considerada como responsabilidade social e ética, principalmente quando relacionada aos serviços públicos (PISCO, 2006; RIGHI; SCHIMIDT; VENTURINI, 2010).

Para Vasconcellos (2002), a principal diferença na definição de qualidade na prestação de serviços está na subjetividade e na dificuldade de definição de qualidade. Cada cliente possui uma determinada percepção sobre qualidade e, muitas vezes, esta diferença resulta até mesmo do emocional do cliente no momento da prestação do serviço.

É imprescindível o entendimento que o conceito de qualidade vai depender da percepção de cada um em função da sua cultura ou do grupo que se irá considerar. Pode-se atestar uma boa qualidade de serviço, levando em consideração as percepções dos clientes, que devem exceder suas expectativas. Não basta compreender a importância de se fornecer serviços com excelente qualidade, é preciso que haja um processo contínuo de monitoramento das percepções dos clientes sobre a qualidade do serviço, detectar as não conformidades e adotar medidas de aperfeiçoamento (BERRY; PARASURAMAN; ZEITHAML, 1990; CERQUEIRA, 1994).

A atenção primária deve atuar como um filtro inicial, resolvendo a maior parte das necessidades de saúde dos usuários e ordenando a demanda por serviços de maior complexidade, organizando os fluxos da continuidade da atenção ou do cuidado. Este papel essencial da atenção primária, tanto na resolução dos casos, quanto no referenciamento do usuário para outros níveis de atenção de maior complexidade (MENDES, 2010). 
Quando questionados acerca da satisfação em relação aos serviços de saúde que foram encaminhados a partir das Unidades de Saúde da Família, os usuários também se posicionaram de maneira positiva. Entre os serviços que foram abordados no questionário e citados pelos usuários estavam o Hospital Regional Inácio de Sá (HRIS), Unidade Pernambucana de Atenção Especializada (UPAE), Centro de Atenção Psicossocial (CAPS) e Policlínica. A tabela 6 mostra os resultados percentuais por tipo de unidade.

Tabela 6 - Satisfação dos usuários em relação ao atendimento recebido nos serviços de saúde no município de Salgueiro- PE quando referenciados pelas ESF, 2016.

\begin{tabular}{lcccc}
\hline $\begin{array}{l}\text { Qual o nível de satisfação } \\
\text { com o atendimento recebido } \\
\text { nesse serviço? }\end{array}$ & & & Serviços & \\
& & & & \\
& HRIS & UPAE & CAPS & Policlínica \\
\hline Insatisfeito & 16,67 & 10,00 & 0 & 0 \\
Pouco satisfeito & 41,66 & 35,00 & 0 & 0 \\
Satisfeito & 16,67 & 0 & 0 & 28,57 \\
Muito satisfeito & 25,00 & 55,00 & 100,00 & 71,43 \\
Total & 100 & 100 & 100 & 100 \\
\hline
\end{tabular}

Fonte: Pesquisa direta, 2016.

Em todos os serviços que foram abordados pela pesquisa, constatou-se que a maioria dos usuários considerou como "muito satisfeito" o atendimento recebido, como o caso da UPAE (55\%), CAPS (100\%) e Policlínica (71,43\%). Já no HRIS, o maior percentual declarou estar pouco satisfeito ou insatisfeito com o atendimento $(58,33 \%)$. Os usuários responderam a pesquisa com base na sua percepção geral sobre os serviços.

Para garantia de melhoria da qualidade da assistência dos serviços de saúde é importante que sejam admitidas as diretrizes do SUS, a universalidade, a integralidade, e a resolutividade, além do controle social, o acolhimento e humanização. Deve-se considerar ainda, a satisfação do usuário, sendo esses componentes importantes para reorganização dos serviços (MACHADO; NOGUEIRA, 2008; MATOS et al., 2002).

As pesquisas de satisfação vêm apresentando resultados muito positivos em relação aos serviços de saúde. Esse retrato é visto amplamente nas pesquisas com perguntas fechadas. Para o usuário, a definição de satisfação vai depender de como o mesmo se ver em relação ao serviço de saúde e do papel que o mesmo tem na sociedade (HIGAKI; KOTAKA; PACHECO, 1997; WILLIAMS, 1994). 


\section{Considerações finais}

O estudo permitiu observar considerações importantes em relação à satisfação dos usuários. Os resultados mostraram que o sexo feminino, baixa escolaridade, renda e cor parda foram características independentemente associadas ao maior uso e à maior satisfação com os serviços de saúde.

Nesta análise, a avaliação dos serviços de saúde do município foi considerada positiva pelos usuários, embora tenham sido observados números altos de insatisfação em relação ao tempo de espera para atendimento e acolhimento. A visão do usuário é de extrema importância ao se dimensionar o reflexo das ações que vêm sendo desenvolvidas no setor saúde, e serve como vetor de direcionamento e planejamento do serviço.

As pesquisas de satisfação retratam resultados temporários e que precisam estar sempre em evidência, pois refletem as condições dos serviços que são oferecidos aos usuários, bem como a avaliação das políticas públicas de saúde, sendo necessário um aprimoramento contínuo. A avaliação da qualidade dos serviços de saúde disponíveis permite que as organizações conheçam detalhadamente o seu desempenho, considerando os critérios de excelência. Não se trata apenas de organizar os serviços para a demanda, mas de ordená-los para as necessidades dos usuários (BONATO, 2011).

Apontam-se como fragilidades da pesquisa a aplicação dos questionários somente nas Unidades de Saúde da Família localizada na zona urbana, além da análise ser apenas quantitativa. Futuros trabalhos de análise qualitativa devem ser realizados na intenção de refutar ou corroborar as relações demonstradas e identificar um panorama de todo o município através da expansão da pesquisa para todas as Unidades de Saúde da Família. A constante avaliação da qualidade do serviço seria de grande valia para um acompanhamento do desempenho dessa estratégia, bem como a avaliação das ações de melhoria impostas ao longo do tempo.

Recomenda-se a realização e aprofundamento de metodologias de qualidade para os serviços de saúde; capacitação efetiva de recursos humanos como fator essencial 
de qualidade institucional e a incrementação de ações de controle e avaliação da qualidade dos serviços públicos de saúde.

\section{Referências}

ANDRADE, M. V. et al. A equidade na cobertura da Estratégia Saúde da Família em Minas Gerais, Brasil. Cad. Saúde Pública, Rio de Janeiro, 31(6):1175-1187, jun, 2015a. Disponível em: <http://www.scielo.br/pdf/csp/v31n6/0102-311X-csp-31-6- 1175.pdf>. Acesso em 06 de junho de 2015.

ANDRADE, M. V. et al. Atenção Primária à Saúde - A "menina dos olhos" do SUS: sobre as representações sociais dos protagonistas do Sistema Único de Saúde. Cad. Saúde Pública, Rio de Janeiro, 31(6):1175-1187, jun, 2015b. Disponível em:

<http://www.scielo.br/pdf/csc/v16s1/a20v16s1.pdf >. Acesso em 09 de julho de 2015.

ASSUNÇÃO, R. S. Fatores condicionantes da qualidade do sistema de saúde na visão dos médicos e da população do Recife. 2006. 131f. Dissertação (Mestrado em Engenharia de Produção) - Universidade Federal de Pernambuco, Recife, 2006.

BESERRA, L. S. C.; COSTA, F. B. C.; OLIVEIRA, C. J. Participação popular: A visão do conselho local de saúde do Centro de saúde da família Carlos Ribeiro. Sociedade em Debate, 20(2): 145-171, 2014. Disponível em:

<http://www.rle.ucpel.tche.br/index.php/rsd/article/view/960/742>. Acesso em 09 de julho de 2015.

BONATO, V. L. Gestão de qualidade em saúde: melhorando assistência ao cliente. O Mundo da Saúde, São Paulo: 35(5):319-331; 2011. Disponível em:

$<$ http://bvsms.saude.gov.br/bvs/artigos/gestao_qualidade_saude_melhorando_assistenci a_cliente.pdf $>$. Acesso em 05 de julho de 2015.

BÓS, A. M. G.; BÓS A. J. G. Determinantes na escolha entre atendimento de saúde privada e pública por idosos. Rev. Saúde Publica; 38(1):113-120, 2004. Disponível em: < http://www.scielo.br/scielo.php?pid=S0034- $\quad$ 89102004000100016\&script=sci_abstract $>$. Acesso em 10 de março de 2016.

BRASIL. Conselho Nacional de Saúde. Resolução no 466, de 12 de dezembro de 2012. Diário Oficial da União, nº 12, seção 1, p. 59-62, 13 de junho de 2013.

BRASIL. Ministério da Saúde. Núcleo Técnico da Politica Nacional de Humanização. Humaniza SUS. Política Nacional de Humanização: a humanização como eixo norteador das praticas de atenção e gestão em todas as instancias do SUS. Brasília: Ministério da Saúde, 2004. 
.MINISTÉRIO DA SAÚDE. Portaria no $\mathbf{2 . 4 8 8}$, de 21 de outubro de 2011. Aprova a Política Nacional de Atenção Básica, estabelecendo a revisão de diretrizes e normas para a organização da Atenção Básica, para a Estratégia Saúde da Família (ESF) e o Programa de Agentes Comunitários de Saúde (PACS).

CASTRO, H. C. O et al. A Satisfação dos Usuários com o Sistema Único de Saúde (SUS). Sociedade em Debate, Pelotas, 14(2): 113-134, jul.-dez./2008. Disponível em:

$<$ http://www.revistas.ucpel.tche.br/index.php/rsd/article/view/375/332>. Acesso em 16 de agosto de 2015.

CERQUEIRA, J. P. de et al. Iniciando os conceitos de qualidade total. Biblioteca Pioneira de Administração e Negócios. São Paulo: Pioneira, 1994. Série Qualidade Brasil.

CHAGAS, H. M. A.; VASCONCELlOS, M. P. C. Quando a porta de entrada não resolve: análise das unidades de saúde da família no município de Rio Branco, Acre. Saúde Soc. São Paulo, v.22, n.2, p.377-388, 2013. Disponível em:

$<$ http://www.revistas.usp.br/sausoc/article/view/76438/80151>. Acesso em 01 de junho de 2016.

COELHO, J. S. Construindo a Participação Social no SUS: um constante repensar em busca de equidade e transformação. Saúde Soc. São Paulo, v.21, supl.1, p.138-151, 2012. Disponível em: 〈http://www.scielo.br/pdf/sausoc/v21s1/12.pdf >. Acesso em 06 de agosto de 2015.

CORRADI, E. M. O programa de saúde da família sob a ótica do usuário. Cadernos da Escola de Saúde Enfermagem; n 1, julho, 2014. Disponível em:

<file:///C:/Users/Elder/Downloads/8-32-1-PB.pdf>. Acesso em 09 de junho de 2016.

CORRÊA, A. C. P.; et al. Acesso a serviços de saúde: olhar de usuários de uma unidade de saúde da família. Rev Gaúcha Enferm. Porto Alegre, set; 32(3):451-7; 2011. Disponível em: <http://seer.ufrgs.br/RevistaGauchadeEnfermagem/article/view/16593 >. Acesso em 06 de junho de 2016.

COSTA, M. F. L.; LOYOLA, A. I. Fatores associados ao uso e à satisfação com os serviços de saúde entre usuários do Sistema Único de Saúde na Região Metropolitana de Belo Horizonte, Estado de Minas Gerais, Brasil. Epidemiol. Serv. Saúde, Brasília, 17(4):247-257, out-dez 2008. Disponível em:

$<$ http://scielo.iec.pa.gov.br/scielo.php?script=sci_arttext\&pid=S1679- 49742008000400002 >. Acesso em 10 de março de 2016.

SILVA JUNIOR, G. B. DIAS, E. R. Avaliação da satisfação dos usuários de um serviço de saúde público-privado no nordeste do brasil e a judicialização da saúde. R. Dir. sanit., São Paulo v.17 n.2, p. 13-29, jul./out. $2016 . \quad$ Disponível em: < https://www.revistas.usp.br/rdisan/article/view/122303>. Acesso em: 10 de março de 2016.

ESPERIDIÃO. M; TRAD LAB. - Avaliação de satisfação de usuários. Ciênc Saúde Coletiva; (S10); 303-12; 2005. Disponível em: 
<http://www.scielosp.org/pdf/csc/v10s0/a31v10s0.pdf>. Acesso em 05 de julho de 2015.

FADEL, M.A.V.; FILHO, G. I. R. Percepção da qualidade em serviços públicos de saúde: um estudo de caso. RAP - RIO DE JANEIRO 43(1):07-22, JAN./FEV. 2009. Disponível em: < http://www.scielo.br/pdf/rap/v43n1/a02v43n1.pdf >. Acesso em 09 de junho de 2015.

FARIAS LO, MELAMED C. Segmentação de mercados da assistência à saúde no Brasil. Cienc Saude Coletiva; 8(2):585-98; 2003. Disponível em:

<http://www.scielosp.org/pdf/csc/v8n2/a19v08n2>. Acesso em 16 de agosto de 2015.

FERRO, D. et al. Satisfação do paciente em um programa educativo para automonitorização da glicemia capilar no domicilio. Rev. Eletr. Enf. 2015 jan./mar.;17(1):37-42. Disponível em:

<https://www.fen.ufg.br/fen_revista/v17/n1/pdf/v17n1a04.pdf >. Acesso em 09 de junho de 2015 .

FIGUEIRÓ A. C., FRIAS P. G., NAVARRO. Avaliação em Saúde: Bases Conceituais e Operacionais. Rio de Janeiro: MedBook. P.196, 2010.

FONTENELE, A. M. M.; ALVES, S. M. A.; CÂMARA, J. T. Avaliação do programa nacional de atenção à hipertensão e diabetes: satisfação dos usuários de uma unidade básica de saúde em Caxias - MA. Cad. Pesq., São Luís, v. 19, n. 1, jan./abr. 2012.

Disponível em:

<http://www.periodicoseletronicos.ufma.br/index.php/cadernosdepesquisa/article/view/ 936/624>. Acesso em 05 de outubro de 2015.

GOUVEIA, G. C. et al. Satisfação dos usuários com a assistência de saúde no estado de Pernambuco, Brasil, 2005. Ciência \& Saúde Coletiva, 16(3); 1849-1861, 2011.

Disponível em: <http://www.scielo.br/pdf/csc/v16n3/20.pdf>. Acesso em 16 de agosto de 2015.

GOUVEIA, G. C. et al. Satisfação dos usuários do sistema de saúde brasileiro: fatores associados e diferenças regionais. Rev Bras Epidemiol; 12(3): 281-96; 2009.

Disponível em: 〈http://www.scielo.br/pdf/rbepid/v12n3/01.pdf>. Acesso em 05 de julho de 2015.

GRDEN, C. R. B.; WEISE, T.; RECHE, P. M.; BORGES, P. K. O.; CABRAL, L. P. A. Características sociodemográficas e de acesso de longevos aos serviços de saúde.

Cienc. Cuid. Saude, Out/Dez; 14(4):1505-1512, 2015. Disponível em:

<http://eduem.uem.br/ojs/index.php/CiencCuidSaude/article/view/24985/pdf >. Acesso em 05 de junho de 2016.

GUEDES, D.G.M.; GARCIA, T.R. Atendimento nos serviços do Sistema Único de Saúde e satisfação do usuário: estudo no município de Campina Grande (PB). Saúde em Debate, Rio de Janeiro, v. 25, n. 59, p. 40-9, 2001. Disponível em:

<http://search.bvsalud.org/cvsp/resource/pt/lil-337034 >. Acesso em 10 de março de 2016. 
KOTAKA F.; PACHECO M. L.; HIGAKI Y. Avaliação pelos usuários dos hospitais participantes do programa de qualidade hospitalar do Estado de São Paulo, Brasil.

Revista de Saúde Pública; 31(2):171-177, 1997. Disponível em:

<http://www.scielosp.org/pdf/rsp/v31n1/2188.pdf>. Acesso em 16 de agosto de 2015.

LIMA, C. M. F.; MATOS, D.L; CAMARANO A. A. Evolução das desigualdades sociais em saúde entre idosos e adultos brasileiros: um estudo baseado na Pesquisa Nacional por Amostras de Domicílios (PNAD 1998, 2003). Ciência \& Saúde Coletiva 2006;11:941950, 2006. Disponível em:

<http://www.redalyc.org/articulo.oa?id=63011416 >. Acesso em 10 de março de 2016.

LIMA, L.; PIRES, D. E. P.; FORTE, E. C. N.; MEDEIROS, F. Satisfação e insatisfação no trabalho de profissionais de saúde da atenção básica. Escola Anna Nery Revista de Enfermagem 18(1) Jan-Mar 2014. Disponível em:

$<$ http://www.scielo.br/pdf/ean/v18n1/1414-8145-ean-18-01-0017.pdf $>$. Acesso em $09 \mathrm{de}$ junho de 2015.

MACHADO N. P., NOGUEIRA L. T. Avaliação da satisfação dos usuários de serviços de Fisioterapia. Rev Bras Fisioter; 12(5):401-8; 2008. Disponível em:

<http://www.scielo.br/pdf/rbfis/v12n5/a10v12n5.pdf>. Acesso em 05 de julho de 2015.

MARTINS, A. L. X.; SANTOS, S. M. R. O Exercício do Controle Social no Sistema Único de Saúde: a ótica do enfermeiro como membro do Conselho Local de Saúde. Saúde Soc. São Paulo, v.21, supl.1, p.199-209, 2012. Disponível em:

<http://www.scielo.br/pdf/sausoc/v21s1/17.pdf > . Acesso em 09 de julho de 2015.

MATOS D. L.; LIMA-COSTA M. F., GUERRA H. L., MARCENES W. Projeto

Bambuí: avaliação de serviços odontológicos privados, públicos e de sindicato. Rev Saude Publica. 36(2):237-43; 2002. Disponível em:

<http://www.scielo.br/pdf/rsp/v36n2/9217.pdf>. Acesso em 05 de julho de 2015.

MENDES, E. V. As redes de atenção à saúde. Ciênc. Saúde Coletiva, v. 15, n. 5, p.22972305, 2010. Disponível em: 〈http://www.scielo.br/pdf/csc/v15n5/ v15n5a05.pdf>. Acesso em 05 de junho de 2016.

MITRE, S. M.; ANDRADE, E. I. G.; COTTA, R. M. M. Avanços e desafios do acolhimento na operacionalização e qualificação do Sistema Único de Saúde na Atenção Primária: um resgate da produção bibliográfica do Brasil. Ciência \& SaúdeColetiva, 17(8):2071-2085, 2012. Disponível em:

<http://www.scielo.br/pdf/csc/v17n8/18.pdf>. Acesso em 03 de junho de 2015.

MURRAY CJL, KAWABATA K, VALENTINE N. People's Experience Versus People's Expectations. Satisfaction measures are profoundly influenced by expectations, say these WHO researchers. Health Affairs; 20(3): 21-4; 2001.

Disponível em: <http://content.healthaffairs.org/content/20/3/21.full>. Acesso em 05 de julho de 2015. 
PAIM J. S. Avaliação em saúde: dos modelos teóricos à prática na avaliação de programas e sistemas de saúde. Cad. Saúde Pública, Rio de Janeiro, 22(1):228-235, jan, 2006. Disponível em: < http://www.scielo.br/pdf/csp/v22n1/27.pdf>. Acesso em 09 de junho de 2015.

PAIM, C. R. P.; CICONELLI, R. M. Auditoria de avaliação da qualidade dos serviços de saúde. RAS _ Vol. 9, No 36 - Jul-Set, 2007. Disponível em:

<http://www.institutoconscienciago.com.br/blog/wp-content/uploads/2012/11/Texto

Auditoria.pdf>. Acesso em 09 de junho de 2015.

PARENTE, J. Varejo no Brasil: gestão e estratégia. São Paulo: Atlas, 2000.

PIOVESAN, A.; LIMA, L. D.; OTTOBELlI, C.; GERMANI, A. R. M. CONTROLE SOCIAL: FERRAMENTA NO PROCESSO DE CONSTRUÇÃO DO SISTEMA

ÚNICO DE SAÚDE. Revista de Enfermagem Frederico Westphalen; v.4e5 n.4e5 p.89105; 2014. :

<http://www.revistas.fw.uri.br/index.php/revistadeenfermagem/article/view/1137>. Acesso em 03 de junho de 2016.

PIRES, M. R. G. M. et al. Oferta e demanda por média complexidade/SUS: relação com atenção básica. Ciência \& Saúde Coletiva, Rio de Janeiro, v.15, n. 1, p. 1009-1019, 2010. Disponível em: < http://www.scielo.br/scielo.php?script=sci_arttext\&pid=S141381232010000700007>. Acesso em 05 de junho de 2016.

PISCO, L. A. A avaliação como instrumento de mudança. Cien Saude Colet; 11(3):566-568, 2006. Disponível em: <http://www.scielo.br/pdf/csc/v11n3/30971.pdf>. Acesso em 05 de julho de 2015.

RAMOS D. D., LIMA M. A. D. S. Acesso e acolhimento aos usuários em uma unidade de saúde de Porto Alegre, Rio Grande do Sul, Brasil. Cad Saude Publica; 19(1):27-34; 2003. Disponível em: <http://www.scielosp.org/pdf/csp/v19n1/14902.pdf>. Acesso em 16 de agosto de 2015.

RIGHI, A. W.; SCHMIDT, A. S.; VENTURINI, J. C. Qualidade em serviços públicos de saúde: Uma avaliação da estratégia saúde da família. Revista Produção Online, v.10, n.3, p. 649-669, set, 2010. Disponível em:

<http://producaoonline.org.br/rpo/article/view/405>. Acesso em 09 de junho de 2015.

RODRIGUES, R. M.; RAIMUNDO, C. B.; SILVA, K. C. Satisfação dos usuários dos serviços privados de fisioterapia do município de Campos dos Goytacazes/RJ. Perspectiva Online volume $4 . \quad$ Número $14, \quad 2010 . \quad$ Disponível emhttp://www.seer.perspectivasonline.com.br/index.php/revista_antiga/article/view/437/ 47>. Acesso em 09 de junho de 2015.

ROLIM, L. B.; CRUZ, R. S. B. L. C.; SAMPAIO, K. J. A. Participação popular e o controle social como diretriz do SUS: uma revisão narrativa. Saúde em debate, v. 37, n. 96, p. 139147, jan./mar. 2013. Disponível em:

<http://www.scielo.br/pdf/sdeb/v37n96/16.pdf >. Acesso em 10 de junho de 2015. 
SANTOS, M. C. P.; JUNIOR, F. A. T.; LELES, F. A. O fortalecimento do Conselho Estadual de Saúde de Minas Gerais a partir do estabelecimento da Lei Complementar 141/2012. Cad. IberAmer. Direito. Sanit., Brasília, v.2, n.2, jul./dez. 2013. Disponível em: < http://www.cadernos.prodisa.fiocruz.br/index.php/cadernos/article/view/73/117>. Acesso em 05 de outubro de 2015.

SIQUEIRA, D. M. R.; LEÃO, M. M.; DALTRO, O.; BIANCHI, E. GIMENEZ, I. G. A pesquisa e análise de satisfação como ferramenta de gestão do relacionamento com o consumidor. REVISTA FAIPE. Cuiabá, v. 4, n. 1, p. 12-18, jan./jun. 2014. Disponível em: <http://www.revistafaipe.com.br/index.php/RFAIPE/article/view/41/74>. Acesso em 05 de outubro de 2015.

STEVENSON J. F., FLORIN P.,MILLS D. S. \& ANDRADE M. Building evaluation capacity in human service organizations: a case study. Eval Program Plann; 25:233- 43, 2002. Disponível em:

<http://www.sciencedirect.com/science/article/pii/S0149718902000186>. Acesso em 05 de julho de 2015.

SZWARCWALD C. L.; MENDONÇA M. H. M.; ANDRADE C. L. Indicadores de atenção básica em quatro municípios do estado do Rio de Janeiro, 2005: resultados de inquérito domiciliar de base populacional. Cien Saude Colet; 11(3):643-655, 2006. Disponível em: <http://www.scielo.br/pdf/csc/v11n3/30980.pdf>. Acesso em 16 de agosto de 2015.

TRAD, L. A. B.; BASTOS, A.C.S. Santana EM, Nunes MO. Estudo etnográfico da satisfação do usuário do Programa de Saúde da Família (PSF) na Bahia. Cienc Saude Coletiva. 2002;7(3):581-9. Disponível em:

<http://www.scielo.br/pdf/csc/v7n3/13034.pdf >. Acesso em 10 de março de 2016.

TURRINI, R. N. T.; LEBRÃO, M. L.; CESAR, C. L. G. Resolutividade dos serviços de saúde por inquérito domiciliar: percepção do usuário. Cad. Saúde Pública, Rio de Janeiro, 24(3):663-674, mar, 2012. Disponível em:

$<$ http://www.scielo.br/pdf/csp/v24n3/20.pdf > . Acesso em 08 de junho de 2016.

VAITSMAN, J.; ANDRADE, G. R. B. Satisfação e responsividade: formas de medir a qualidade e a humanização da assistência à saúde. Ciência \& Saúde Coletiva; 10(3); 599613, 2005. Disponível em: <http://www.scielo.br/pdf/csc/v10n3/a17v10n3.pdf>. Acesso em 16 de agosto de 2015.

VASCONCELLOS, P. P. de. Desenvolvimento de um modelo de avaliação da qualidade do serviço odontológico. 2002. 91 f. Dissertação (Mestrado em Engenharia de Produção) Centro Tecnológico, Universidade Federal de Santa Catarina, Florianópolis. Disponível em: $<$ https://repositorio.ufsc.br/xmlui/bitstream/handle/123456789/81778/185282.pdf?seque nce $=1 \&$ is Allowed $=y>$. Acesso em 05 de julho de 2015 .

WILlIAMS, B. Patient satisfaction: a valid concept? Social Science and Medicine; 38(4):509-516, 1994. Disponível em: 
<http://www.sciencedirect.com/science/article/pii/027795369490247X>. Acesso em 16 de agosto de 2015.

ZEITHAML, V. A.; PARASURAMAN, A.; BERRY, L. L. Delivering quality service: balancing customer perceptions and expectations. New York: The Free Press, 1990. 226 p.

\section{Como citar este artigo (Formato ABNT):}

CRUZ, Ially L.de C.; PARENTE, Alaine S.; MESQUISTA, Fabíola O.de S.; JERÔNIMO, Aline Silva. Satisfação dos usuários com os serviços do Sistema Único de Saúde - SUS em um município do sertão de Pernambuco. Id on Line Revista ultidisciplinar e de Psicologia, 2017, vol.12, n.39, p.142--163. ISSN: 1981-1179.

Recebido: 15.11 .2017

Aceito: 18.11 .2017 